Tjalling C. Koopmans Research Institute

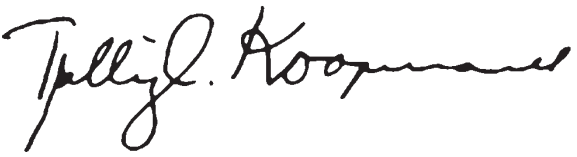

Discussion Paper Series nr: 07-11

\title{
Efficient Communication in the Electronic Mail Game
}

Kris de Jaegher 


\section{Tjalling C. Koopmans Research Institute Utrecht School of Economics \\ Utrecht University}

Janskerkhof 12

3512 BL Utrecht

The Netherlands

telephone $\quad+31302539800$

fax $\quad+31302537373$

website www.koopmansinstitute.uu.nl

The Tjalling C. Koopmans Institute is the research institute and research school of Utrecht School of Economics.

It was founded in 2003, and named after Professor Tjalling C. Koopmans, Dutch-born Nobel Prize laureate in economics of 1975.

In the discussion papers series the Koopmans Institute publishes results of ongoing research for early dissemination of research results, and to enhance discussion with colleagues.

Please send any comments and suggestions on the Koopmans institute, or this series to s.mook@econ.uu.nl

ontwerp voorblad: WRIK Utrecht

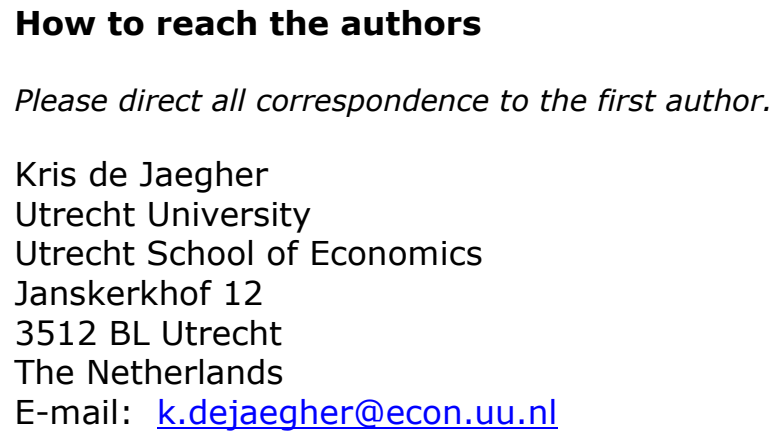

This paper can be downloaded at: http://www.koopmansinstitute.uu.nl 
Utrecht School of Economics

Tjalling C. Koopmans Research Institute

Discussion Paper Series 07-11

\title{
Efficient Communication in the Electronic Mail Game
}

\author{
Kris de Jaeghera \\ aUtrecht School of Economics \\ Utrecht University
}

January 2007

\begin{abstract}
The literature on the electronic mail game shows that players' mutual expectations may lock them into requiring an inefficiently large number of confirmations and confirmations of confirmations from one another. This paper shows that this result hinges on the assumption that, with the exception of the first message, each player can only send a message when receiving an immediately preceding message. We show that, once this assumption is lifted, equilibria involving confirmations of confirmations no longer pass standard refinements of the Nash equilibrium, and are no longer evolutionary stable.
\end{abstract}

Keywords: Electronic Mail Game, Efficient Communication, Grounding, Equilibrium Refinements, Evolutionary Stability

JEL classification: C70, D72

Acknowledgements

I would like to thank an anonymous referee, Stephanie Rosenkranz, for helpful comments. Any remaining errors are my own. 


\section{INTRODUCTION}

How many messages do people need to exchange before they can agree to undertake a mutually beneficial joint endeavor? At first sight, the answer given by game theory would seem to be found in Rubinstein's (1989) analysis of the electronic mail game (henceforth EMG, see Section 2 below for this game in its most general form), and would follow the lines of the following story. Rowena, a game theorist, finds out that there is a showing of A Beautiful Mind at the local cinema, and wants to take a fellow game theorist, Colin, on a first date to see this movie. Rowena sends Colin a message, inviting him to go to the movie. However, given that the possibility that her message got lost, Rowena worries that she will end up alone at the cinema. Moreover, Rowena runs little risk when staying home. This is why she only envisages going to the cinema if she receives a confirmation from Colin. But Colin, who is equally worried about showing up by himself, is now again concerned that Rowena did not hear his confirmation, and requires a confirmation of his confirmation. Rowena now again wants a confirmation of her confirmation of his confirmation. And so on, ad infinitum. Frustratingly then, if Rowena and Colin reason like game theorists, it seems they never meet each other on a date. ${ }^{1}$ However, as shown in this paper, game theory can in fact be used to show that Rowena will meet Colin at the cinema most of the time, and that at most a single confirmation will be sent.

To see the reason for the unintuitive result reflected in the story above, it should be noted that Rubinstein (1989) introduced the EMG into the literature not to model how people trying to achieve coordinate action communicate, but rather to show that approximate common knowledge is very different from common knowledge (see Section 3 of our paper for a review of this result). Concretely, in terms of the story above, Rubinstein assumes that, with the exception of the first message sent by Rowena, all messages take the form of unambiguous proofs of receipt, and that these proofs of receipt are automatically sent back and forth between Rowena and Colin, until a proof of receipt gets lost. With each new proof of receipt received, the recipient achieves a higher level of interactive knowledge ("I know that you know that I know..."). For a vanishing probability that proofs of receipt gets lost, the message exchange would therefore seem to approach a situation of common knowledge. However, even with an infinitesimally small probability that a proof of receipt gets lost, coordinated action does not take place.

As pointed out by Binmore and Samuelson (2001), the EMG can in fact be used to analyze the communication of people who try to achieve coordinated action, but some modifications in the assumptions are then called for (see Section 4 below for a review of their results). A first modification to the EMG that they make is to assume that communication is not automatic, but strategic. A second modification that they make is to assume that communication is costly, in that both sending a message, and paying attention to a message is costly. However, Binmore and Samuelson maintain the assumption that, after the first message has been sent, communication takes the form of unambiguous proofs of receipt. The fact that communication is costly puts a cap on the number of messages that will be shuttled back and forth. The fact that communication is voluntarily assures the existence of more intuitive equilibria, where either a single message by Rowena suffices, or where Rowena contents herself with a

\footnotetext{
${ }^{1}$ A similar result was shown by Halpern and Moses (1984) in the distributed systems literature, where this problem is known as the coordinated attack problem.
} 
single confirmation by Colin. These are also the Pareto-efficient equilibria. However, Pareto inefficient equilibria also exist. The reason for this is that the players' mutual expectations can lock them into requiring a large number of confirmations and confirmations of confirmations from one another. While an intuitive result is now at least possible, the possibility of an unintuitive result is thereby maintained.

The current paper takes this analysis one step further by lifting the assumption that messages take the form of proofs of receipt. In the game analyzed here, players are able to send a message even when they did not receive an immediately preceding message. While Nash equilibria where players send confirmations and confirmations of confirmations continue to exist, only the Pareto efficient equilibria survive standard refinements of the Nash equilibrium (Section 5). There are two basic intuitions for this result.

The first intuition is that Pareto inefficient equilibria are based on threats that are not credible (backward induction). In our modified game, it is possible to send a 'confirmation' even when no message was received. We refer to such a message as a false acknowledgement. The receiver of a confirmation will detect it to be false when he or she did not send the message that the false acknowledgement claims to be confirming. The receiver can only stop a false acknowledgement from being sent by not showing up at the cinema when detecting a confirmation to be false. However, this does not suffice as a punishment. The receiver should moreover reply to the false acknowledgement, thus acting in the same manner as when the sender's confirmation was not detected to be false. Otherwise, when not receiving a reply to his or her false acknowledgement, the sender gets a strong hint that he or she was caught cheating. He or she then stays at home, avoids the cost of being stood up, and thereby escapes much of the punishment. But from the receiver's perspective, choosing the safe option of staying home and still sending a costly message is never a best response. The receiver's threat of punishing detected false acknowledgements is therefore not credible.

The second intuition for eliminating equilibria where a large number of messages is sent looks at how Colin should interpret a detected false acknowledgement (forward induction). When there is no good movie on, given that in equilibrium Colin stays at home when not receiving any messages, Rowena has no reason to send any messages. Also, when there is a good movie on, but if Rowena is planning not to go to the cinema, she has no reason to send any messages. It follows that Colin should not interpret a detected false acknowledgement sent by Rowena as an act of cheating, but on the contrary as an attempt to still achieve mutually beneficial coordinated action.

Section 6 shows that the latter intuition also applies in a modified version of the EMG, where players at each stage of the game can choose between sending proofs of receipt, and messages that do not prove receipt. In a Nash equilibrium where many proofs of receipt are sent back and forth, by a forward induction argument, when one player receives a message even though he or she did not send a proof of receipt, then this player should interpret this as a repair message, i.e. as an attempt by the other player to still achieve coordinated action. But if our player responds positively to such a repair message, then the other player no longer has any incentive to send the proof of receipt immediately preceding this repair message.

Section 7 shows that the equilibrium refinements have an evolutionary underpinning. The Pareto inferior equilibria where confirmations of confirmations are sent are not evolutionary stable. The paper ends with a conclusion in Section 8, where further directions for research are also suggested. 


\section{GENERIC ELECTRONIC MAIL GAME}

For clarity of exposition, let us start by describing the most straightforward aspects of the EMG. There are two states of nature, state $a$ of $b$, and the two players 1 and 2 can choose from two actions, namely action $A$ and action $B$. Figure 1 denotes the benefits that players get as a function of the states (game $G_{a}$ is played in state $a$, game $G_{b}$ in state $b$ ), and as a function of the actions that they take. It is assumed that

$$
L>M>0
$$

meaning that the cost of doing $B$ when the other player is doing $A$ is relatively large. Game $G_{a}$ occurs with probability $(1-p)$, game $G_{b}$ with probability $p$. It is assumed that

$$
(1-p)>1 / 2
$$

meaning that $G_{b}$ is occurs less often.

A useful fiction to formulate verbal intuitions for our results is that either there is a good movie on television (the more likely state $a$ ), or at the cinema (the less likely state $b$ ) (the probability that there are only bad movies at the cinema and on television is negligible). Action $A$ means staying home, action $B$ means going to the cinema. Rowena (player 1) and Colin (player 2) obtain their highest benefit $M$ if they see a good movie together. Seeing a bad movie together yields payoff 0 . Staying at home alone also yields payoff 0 , whether or not there is a good movie on. Going to the cinema alone, whether or not there is a good movie on, causes a $\operatorname{cost} L$.

A

\begin{tabular}{|l|l|}
\hline$(M, M)$ & $(0,-L)$ \\
\hline$(-L, 0)$ & $(0,0)$ \\
\hline
\end{tabular}

$G_{a}\left(\left\{r_{1}^{1}=0\right\}\right.$, probability $\left.(1-p)>1 / 2\right)$
A

\begin{tabular}{|l|l|}
\hline$(0,0)$ & $(0,-L)$ \\
\hline$(-L, 0)$ & $(M, M)$ \\
\hline
\end{tabular}

$G_{b}\left(\left\{r_{1}^{1}=1\right\}\right.$, probability $\left.p\right)$

Fig. 1. The electronic mail game

A first complicating fact in the model is that, first, only one player (player 1, Rowena) knows the state of nature. It follows that, for players to obtain payoff $M$ in both states, the informed player needs to inform the uninformed player about the state of nature. We will focus on equilibria where player 1 informs player 2 in state $b$ (good movie on at the cinema). A second complicating factor is that communication is assumed to be faulty, in that messages may get lost. Because of this fact, and because taking action $B$ by oneself is costly, after having sent a message, player 1 may only 
want to take action $B$ when player 2 confirms receipt of her message. For the same reason, player 2 may want to receive a confirmation that his confirmation was received. Player 1 again may want to receive a confirmation that player 2 received her confirmation of the fact that she received his confirmation of her initial message. And so on, ad infinitum. Formally, after player 1's first message, each player in turn gets the opportunity to confirm receipt of the other player's last message (cf. Rubinstein, 1989). A third complicating factor is that, following Binmore and Samuelson (2001) players are modeled as not automatically receiving messages, but can only receive a message if they are paying attention at the time the message is sent. Concretely, at the start of the game, players decide on whether or not to keep a channel open for receiving messages at each relevant future stage of the game. A fourth complicating factor is that, again following Binmore and Samuelson (2001), both sending messages and paying attention to messages is assumed to be costly.

Formally, as summarized in Figure 2, players make their attention paying decisions at stage 0 of the game. Nature decides about whether state $a$ or $b$ occurs at stage 1 . Players make their signaling decisions at stages $\{2,3, \ldots, z\}$; Nature each time simultaneously decides whether these messages arrive. $z$ denotes the last stage at which any message can be sent. Players make their action decisions $(A$ or $B)$ at stage $(z+1)$, and finally obtain their payoffs at stage $(z+2)$.

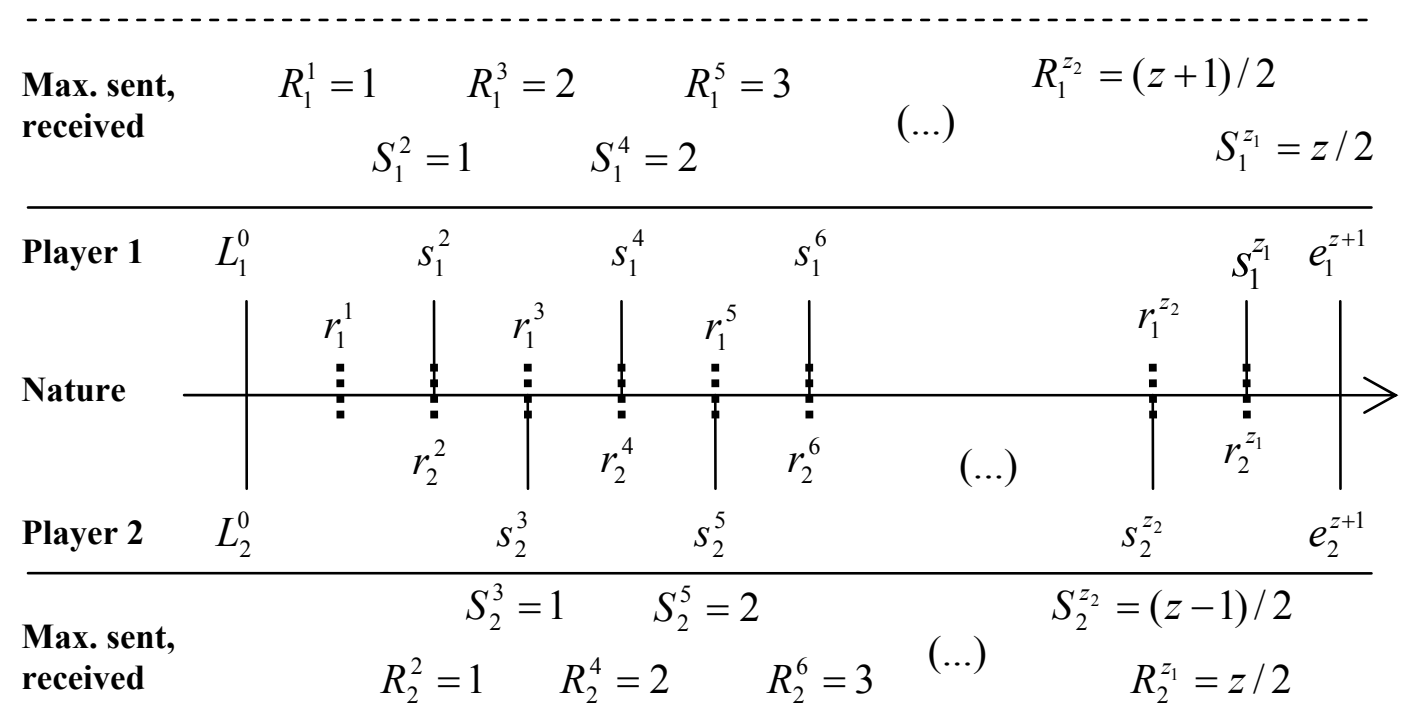

Fig. 2 Attention paying, receipt, signaling, and action decisions

The players' decision at different stages of the game are summarized in the part of Figure 2 between the two solid horizontal lines (the part of the figure below and above these solid lines will be explained later). We start with the signaling and receipt decisions from stages 1 to $z$. Player $i$ (with $i \in\{1,2\}$ ) makes his signaling decisions at stages $t$, where $t \in\left\{(i+1),(i+3),(i+5), \ldots, z_{i}\right\} . z_{i}$ denotes the last stage at which player $i$ can send a message (with either $z_{i}=z$, or $z_{i}=(z-1)$; Figure 2 shows the case where $z_{1}=z$ ). A typical signaling decision is denoted $s_{i}^{t}$, where $s_{i}^{t} \in[0,1]$. In event 
$\left\{s_{i}^{t}=1\right\}$, player $i$ sends the message at stage $t$ and in event $\left\{s_{i}^{t}=0\right\}$, player $i$ does not send the message. The cost incurred by a player for each message sent is $d$.

Given that player $i$ sends messages at stages $t=\left\{(i+1),(i+3),(i+5), \ldots, z_{i}\right\}$, Nature decides at these same stages whether or not to let player $j$ (where $j \neq i$ ) receive the message sent by player $i$. An individual receipt decision by Nature is denoted as $r_{j}^{t}$, where $r_{j}^{t} \in[0,1]$. In event $\left\{r_{j}^{t}=1\right\}$, player $j$ receives player $i$ 's message sent at stage $t$; in event $\left\{r_{j}^{t}=0\right\}$, player $j$ does not receive player $i$ 's message sent at stage $t$. It is assumed that $\pi\left(\left\{r_{j}^{t}=1\right\} \mid\left\{s_{i}^{t}=1\right\}\right)=\psi \quad$ and $\pi\left(\left\{r_{j}^{t}=0\right\} \mid\left\{s_{i}^{t}=1\right\}\right)=(1-\psi)$, where $\pi(\{G\}\{F\})$ denotes the probability of event $G$ occurring given that event $F$ has occurred. Also, $\pi\left(\left\{r_{j}^{t}=1\right\} \mid\left\{s_{i}^{t}=0\right\}\right)=0$. Player $j$ thus never receives a message when none was sent by the other player. Put otherwise, false negatives can occur, but false positives cannot. It is assumed that

$$
\psi M-(1-\psi) L>0
$$

meaning that in state $b$, player 1 prefers to play $B$ after having sent a single message if this implies that player 2 plays $B$ when receiving the message, and plays $A$ otherwise. (3) is a necessary condition for any information transmission from player 1 to player 2 to be possible. As by (1) it is the case that $L>M$, (3) implies that $\psi$ is small.

At stage 1, Nature also decides whether state $a$ or $b$ occurs. Because this makes it easier to denote player 1's information sets and strategies, we model the state that occurs as a decision by Nature on the value of $r_{1}^{1}$, where event $\left\{r_{1}^{1}=0\right\}$ means that at stage 1 (superscript) player 1 (subscript) does not get any message from Nature (state $a$ occurs), and where $\left\{r_{1}^{1}=1\right\}$ means that at stage 1 player 1 gets the message from Nature (state $b$ occurs). These messages from Nature are not noisy.

Having listed the stages at which player $j$ can receive a message, we are now ready to list the attention paying decisions of player $j$. Player $j$ 's decision at stage 0 about the future stages for which he keeps a channel open is denoted by a vector $L_{j}^{0}=\left(l_{j}^{j}, l_{j}^{j+2}, l_{j}^{j+4}, \cdots, l_{j}^{z_{i}}\right)$ where $l_{j}^{t} \in[0,1]$. We say that player $j$ pays attention (respectively does not pay attention) at stage $t$ when $l_{j}^{t}=1$ (respectively when $l_{j}^{t}=0$ ). For each stage paid attention to, a player incurs a cost of $c$. Note that the stages $t=\left\{j,(j+2),(j+4), \ldots, z_{i}\right\}$ in the vector $L_{j}^{0}$ are identical to the stages $t=\left\{(i+1),(i+3),(i+5), \ldots, z_{i}\right\}$ at which player $j$ can receive messages from $i$, with the exception that for $j=1$, the vector includes an attention paying decision for stage 1 . Player 1 therefore only finds out the state of nature when paying attention at stage 1 .

At stage $(z+1)$, players decide on what action to take, modeled for player $i$ ' as a decision to put $e_{1}^{z+1}$ equal to $A$ or $B$. At stage $(z+2)$ the payoffs are obtained. Net of attention paying and signaling costs, these are listed in Figure 1.

Refer to $h^{t}$ as the true history of events up to stage $t$. As players do not observe all events, refer to $h_{i}^{t}$ as player $i$ 's observed history, meaning the sequence of events that player $i$ has been able to observe up to stage $t$. Denote by $H_{i}^{t}$ the set of all possible observed histories by player $i$ up to stage $t$. An information set for player $i$ is a set of 
possible true histories that may have occurred given player $i$ 's observed history. It is standard to describe player $i$ 's strategy as a plan on what attention paying, signaling and action decisions to make for each information set. For our purposes, however, it is more convenient to describe player $i$ 's strategy as a function of his or her observed histories.

Player $i$ 's strategy then consists of a function $l_{i}^{t}(\phi)$ for all $t \in\left\{i,(i+2), \ldots, z_{j}\right\}$ (where $\phi$ is the empty history), of a function $s_{i}^{t}\left(h_{i}^{t}\right)$ for all $h_{i}^{t} \in H_{i}^{t}$ and for all $t \in\left\{(i+1),(i+3), \ldots, z_{i}\right\}$, and of a function $e_{i}^{z+1}\left(h_{i}^{z+1}\right)$ for all $h_{i}^{z+1} \in H_{i}^{z+1}$. The strategies $l_{i}^{* t}, s_{i}^{* t}$ and $e^{* z+1}$ denote (candidate) equilibrium strategies. $z^{*}$ denotes the last stage at which, in a particular equilibrium, any player sends a message with positive probability (where it need not be the case that $z^{*}=z$ ). $z_{i}^{*}$ denotes the last stage at which, in a particular equilibrium, player $i$ sends a message with positive probability. In all the equilibria that we study, it is either the case that $z_{i}^{*}=z^{*}$ or that $z_{i}^{*}=\left(z^{*}-1\right)$.

To deal with the notational challenge of the model, we will often use short-hand notation to describe player $i$ 's strategy as a function of his observed history. For instance, $s_{i}^{t}\left(\left\{r_{i}^{t-1}=1\right\}\right)=1$ means that player $i$ adopts the strategy of sending the message at stage $t$ for any observed history that includes the event of player $i$ receiving the message at stage $(t-1)$. For further short-hand notation of observed histories, the following notation will be particularly useful. Denote by $R_{i}^{t}$ the maximum number of messages that player $i$ can receive up to stage $t$ (see the values at the bottom and top of Figure 2), and by $\rho_{i}^{t}$ the actual number of times that player $i$ receives a message up to stage $t$. Similarly, denote by $L_{i}^{t}$ and $\lambda_{i}^{t}$ respectively the largest possible and the actual number of messages paid attention to by player $i$ up to stage $t$ (where clearly $L_{i}^{t}=R_{i}^{t}$, but where $\lambda_{i}^{t}$ need not be equal to $\rho_{i}^{t}$ ). $\lambda{ }^{*}{ }_{i}^{t}$ denotes the actual number of messages that player $i$ pays attention to up to stage $t$ in equilibrium. Denote by $S_{i}^{t}$ and $\sigma_{i}^{t}$ respectively the largest possible and the actual number of messages sent by player $i$ up to stage $t$ (see the values at the bottom and top of Figure 2).

As an example of the usefulness of this notation for stating a player's strategy in a concise manner, the expression $e_{i}^{z+1}\left(\left\{\sigma_{i}^{z^{*}}=S_{i}^{z^{*}}\right\} \wedge\left\{\rho_{i}^{z^{*}}=R_{i}^{z^{*}}\right\}\right)=B$ denotes that player $i$ adopts action $B$ upon any observed history such that player $i$ has sent the maximum number of messages that can be sent in equilibrium, and has received the maximum number of messages that can be sent in equilibrium. Another example is $s_{i}^{t}\left(\left\{\sigma_{i}^{t-2}<S_{i}^{t-2}\right\} \vee\left\{\rho_{i}^{t-1}<R_{i}^{t-1}\right\}\right)=0$, which denotes that player $i$ does not send the message at $t$ when, before $t$ (i.e., up to stage $(t-2)$ ), he has not sent the maximum number of messages that he could have sent and/or, when before $t$ (i.e., up to stage $(t-1))$, he has not received the maximum number of messages that he could have received.

We now consecutively treat Rubinstein's game, a slightly modified version of Binmore and Samuelson's game, and finally our own game. The reasons for restating Rubinstein's and Binmore and Samuelson's games are, first, that this allows an easier comparison of their results to the results in this paper, and second, that parts of the 
proofs that are necessary to show these authors' results are also required to prove the results for the variant of the EMG treated in this paper.

\section{RUBINSTEIN'S GAME}

The game treated by Rubinstein can be seen as a variant of the EMG in Section 2, with the following restrictions added:

\section{Definition 1:}

Define as game R the EMG with the following restrictions on the action sets of player $i \in\{1,2\}$ :

(i) $L_{i}^{0}$ such that $\lambda_{i}^{z}=L_{i}^{z}$;

(ii) $s_{i}^{t}\left(\left\{r_{i}^{t-1}=1\right\}\right)=1, s_{i}^{t}\left(\left\{r_{i}^{t-1}=0\right\}\right)=0$ for all $t=\left\{(i+1),(i+3), \ldots, z_{i}\right\}$;

and with the following restriction on the parameters:

(iii) $c=0, d=0$.

In words, in game $\mathrm{R}$, up to stage $z$, players automatically pay attention at all the relevant stages, and messages are automatically shuttled back and forth until a message gets lost. Sending messages and paying attention is costless. Each player can simply be said to observe how many messages he receives. A strategy in game $\mathrm{R}$ is then a mapping from the number of messages received to the action set $\{A, B\}$. Rubinstein's results are summarized in the following proposition:

PROPOSITION 1 (Rubinstein, 1989). In game $R$, there is a unique strict separating Nash equilibrium in which $e_{1}^{*^{z+1}}\left(\left\{r_{1}^{1}=0\right\}\right)=A$. In this equilibrium, it is met for $i \in\{1,2\}$ that $e_{i}^{*_{i}^{z+1}}\left(\left\{\rho_{i}^{z}=R_{i}^{z}\right\}\right)=B, \underset{i}{*_{i}^{z+1}}\left(\left\{\rho_{i}^{z}<R_{i}^{z}\right\}\right)=A$.

Proof:

See Appendix.

The intuition for this result is the following. Colin's uncertainty when not receiving an initial invitation from Rowena to go to the cinema, spills over into uncertainty by Rowena about whether Colin knows that there is a good movie on at the cinema, into uncertainty by Colin whether Rowena knows that Colin knows that there is a good movie on at the cinema, etc., each time leading the player to stay home. In the extreme case where $z=\infty$, players always stay home. ${ }^{2}$

In equilibrium, if player $i$ can receive the message at stage $z$ (case where $\left.z_{i}=(z-1)\right)$, he or she obtains a payoff of $(1-\rho) M+\rho \psi^{z-1} M$. If player $j$ in equilibrium can send the message at stage $z$ (case where $z_{i}=z$ ), he or she obtains a payoff of $(1-\rho) M+\rho \psi^{z-2}[\psi M-(1-\psi) L]$. It is easy to see then that, if the size of $z$ is a matter of design, the Pareto-efficient sizes of $z$ are $z=2$ and $z=3$. Player 2 is

\footnotetext{
${ }^{2}$ The fear of such an outcome seems to underlie the postal acceptance rule in law (Christensen, 2001). This rule says that an offeree who accepts an offer by means of a letter is legally bound by his acceptance letter as soon as he posts it. The offeree cannot claim that he is only legally bound by his acceptance letter when receiving an acknowledgement of receipt of it. The fear is that otherwise, parties would require an ever increasing number of acknowledgements from each other, so that a contract would never be become valid (Christensen, 2001).
} 
best off when a single message is sent from player 1 to player 2 (as player 2 then does not run any risk from playing $B$ ), player 1 is best off when this same message sent from player 1 to player 2 is followed by a confirmation by player 2 (as player 1 then does not run any risk from playing $B$ ).

\section{BINMORE AND SAMUELSON'S GAME}

Binmore and Samuelson (2001) modify the EMG by adding the following realistic assumptions. First, communication is voluntary. Second, paying attention to messages and sending messages is costly. However, just as is the case in game R, communication takes the form of proofs of receipt. Formally:

\section{Definition 2:}

Define as game BS the EMG with the following restriction on the action sets of player $i \in\{1,2\}$ :

(i) $s_{i}^{t}\left(\left\{r_{i}^{t-1}=0\right\}\right)=0$ for all $t=\left\{(i+1),(i+3), \ldots, z_{i}\right\}$.

It should be noted that game BS differs from Binmore and Samuelson's (2001) original game, in that in game BS it is possible for a player to pay attention at stage $\tau$ even though he or she did not pay attention at stage $t$, with $\tau>t$. In Binmore and Samuelson, on the contrary, players decide on an attention span. Our reason for modifying Binmore and Samuelson's game is with a view to the game treated in Section 5.

A first step to analyzing game BS is to show that, in any candidate pure-strategy separating equilibrium of this game, the players' attention paying, signaling and action paying decisions take on a certain form. In particular, Proposition 2 shows that in any such equilibrium, players pay attention at contiguous stages. At the stages where the other player pays attention, each player sends a proof of receipt when receiving a message. Finally, each player only acts when receiving the maximum number of messages that the equilibrium allows him or her to receive.

PROPOSITION 2. For any pure-strategy separating equilibrium in game BS in which $e_{1}^{* z+1}\left(\left\{r_{1}^{1}=0\right\}\right)=A$, for $i \in\{1,2\}$ and for each $z^{*}$ such that $2 \leq z^{*} \leq z$, it is met that

(i) $L \underset{i}{* 0}$ such that $\lambda \underset{i}{* z^{*}}=L_{i}^{z^{*}}=\lambda \underset{i}{* z}, 0 \leq\left|\lambda \underset{i}{* z^{*}}-\lambda \underset{j}{* z^{*}}\right| \leq 1$ (where $j \neq i$ );

(ii) $s_{i}^{* t}\left(\left\{r_{i}^{t-1}=1\right\}\right)=1$ for all $t \in\left\{(i+1),(i+3), \ldots, z_{i}^{*}\right\}$;

(iii) $a_{i}^{*_{i}^{z+1}}\left(\left\{\sigma_{i}^{z^{*}}=S_{i}^{z^{*}}\right\} \wedge\left\{\rho_{i}^{z^{*}}=R_{i}^{z^{*}}\right\}\right)=B, \quad a_{i}^{*^{z+1}}\left(\left\{\sigma_{i}^{z^{*}}<S_{i}^{z^{*}}\right\} \vee\left\{\rho_{i}^{z^{*}}<R_{i}^{z^{*}}\right\}\right)=A$.

Proof:

See Appendix.

By comparing with the definition of game $\mathrm{R}$ (Definition 1), it becomes clear from Proposition 2 that, in any pure-strategy separating equilibrium of game BS where messages are sent with positive probability up to stage $z^{*}$, the players' voluntary attention paying and signaling decisions replicate the automatic decisions for a game $\mathrm{R}$ with a $z$ that happens to be equal to the $z *$ of the given equilibrium in game BS. The assumption that paying attention is costly assures that player $i$ does not pay attention at stages where player $j$ does not send messages; the fact that player $i$ does not pay attention at certain stages in turn assures that player $j$ will not send messages 
at these stages. The difference with game $\mathrm{R}$ is that in game BS, it can be the case that $z^{*}<z$ (where the latter $z$ again refers to game BS).

Assume now that player $i$ has up to stage $z *$ followed the attention and signaling strategies specified in Proposition 2, and faces a player $j$ who does the same. Then it is clear that we again can apply Proposition 1, meaning that in any candidate equilibrium where players send messages with positive probability up to stage $z$, each player only plays $B$ when receiving the maximum number of messages that he or she can receive up to stage $z^{*}$. Put otherwise, if a separating equilibrium exists in game BS where players send messages up to stage $z *$, then the action decisions in this equilibrium completely replicate the action decisions in the unique separating equilibrium of a game $\mathrm{R}$ with a $z$ that happens to be equal to $z *$.

In order to prove that such separating equilibria indeed do exist in game BS, it remains to be shown that the specified attention paying and signaling decisions are mutual best responses. This is shown in Proposition 3 for arbitrarily small $c$ and $d$ (contrary to Binmore and Samuelson (2001), who also consider larger cost levels).

PROPOSITION 3: For sufficiently small $c$ and $d$, for each $z^{*} \in[2,3, \ldots, z]$, in game BS a strict separating Nash equilibrium of the form described in Proposition 2 exists.

Proof:

See Appendix.

The intuition reflected in the first paragraph of this paper continues to apply to game BS. However, the intuition only applies for a given number of messages that the players expect each other to send. It is thus players' mutual expectations that keep them locked into requiring a large number of confirmations, and confirmations of confirmations from each other. Intuitively, if Rowena believes that Colin only goes to the cinema when having received a large number of messages, then Rowena will in turn require confirmations that Colin has received these messages before she is willing to go to the cinema; Colin in turn will want to receive confirmations of the confirmations. This justifies Rowena's beliefs that Colin will only go to the cinema when receiving a certain number of messages, making the circle round.

The advantage in comparison to game $\mathrm{R}$ is that Pareto superior equilibria with $z^{*}=2$ and $z^{*}=3$ now exist, whatever the size of $z$. However, unless the costs of sending messages and/or paying attention to messages are large, these Pareto superior equilibria exist side by side with Pareto inferior equilibria, where a large number of confirmations are sent back and forth. The reason that, contrary to Binmore and Samuelson (2001), we assume arbitrarily small message sending and attention paying costs is that we focus not on signaling and attention costs as a factor limiting the degree of inefficiency, but focus on the use of so-called false acknowledgements (messages sent when no preceding message was received) as a source of efficiency. A first step towards this result is made in Section 5.

\section{ELECTRONIC MAIL GAME WITHOUT PROOFS OF RECEIPT}

The results obtained for games R and BS are based on the assumption that players communicate by means of proofs of receipt. While interlocutors sometimes use special-purpose acknowledgements, e.g. by repeating or paraphrasing the last speaker's utterance, thus providing a proof of understanding, they also use generalpurpose acknowledgements such as "OK!" or "Uh huh." which do not provide any 
proof that the last utterance was understood (Clark, 1996). ${ }^{3}$ We now check whether the same equilibria continue to exist in an EMG where a received message does not provide any proof that the immediately preceding message was received. This means that we study the game set out in Section 2 without any restriction on the action sets.

\section{Definition 3:}

Define as game EMG* the EMG without any restrictions on the action sets.

The particularity of game EMG* is that, at the relevant stages, each player is able to send a message even though he or she did not receive an immediately preceding message. Thus, Colin is able to say 'OK' even though he did not receive a message from Rowena. We refer to the case where a message is sent even though no immediately preceding message was received as the sending of a false acknowledgement. A receiver who receives the message at stage $t$ even though he did not send the message at stage $(t-1)$ is said to detect a false acknowledgement. Formally:

\section{Definition 4:}

(i) If $s_{i}^{t}\left(\left\{r_{i}^{t-1}=1\right\}\right)=1$, then we say that player $i$ sends a true acknowledgement at stage $t$; if $s_{i}^{t}\left(\left\{r_{i}^{t-1}=0\right\}\right)=1$, then we say that player $i$ sends a false acknowledgement at stage $t$ (where $2 \leq t \leq z^{*}$ );

(ii) If $\left\{s_{j}^{t-1}=0\right\}$ and $\left\{r_{i}^{t}=1\right\}$, then we say that player $j$ detects a false acknowledgement at stage $t$ (where $3 \leq t \leq z^{*}$ ).

We now show that the same type of separating Nash equilibria as exist in games $\mathrm{R}$ and BS continue to exist in game EMG*. The crucial feature for the existence of such equilibria is that players punish detected false acknowledgements in a specific manner. Before actually showing the existence of these equilibria, we first derive a result about the form which punishments should take. ${ }^{4}$

PROPOSITION 4. In game EMG*, let player i follow the strategy described in (i)(iii) of Proposition 2, with the addition that $s_{i}{ }_{i}^{t}\left(\left\{\sigma_{i}^{t-2}<S_{i}^{t-2}\right\} \vee\left\{\rho_{i}^{t-1}<R_{i}^{t-1}\right\}\right)=0$ for all $t=\left\{(i+1),(i+3), \ldots, z_{i}^{*}\right\}$. Then:

(i) For $i=2$, player 2's strategy stops player 1 from sending a false acknowledgement at stage 2. For $z=z_{j}^{*}$, player $i$ 's strategy stops player $j$ from sending a false acknowledgements at stage $z_{j}^{*}$. However, player i's strategy described does not stop player j from sending false acknowledgements at any other stages;

\footnotetext{
${ }^{3}$ Many married people will corroborate this observation.

${ }^{4}$ It should be noted that, while the analysis of game R and game BS identified the unique pure-strategy separating equilibria, this is not the case for game EMG*. For instance, as shown in De Jaegher (2005), for certain levels of the attention-paying and message-sending costs, and for certain levels of noise, equilibria exists where the player 1 repeats his message up to a certain maximum number of times when player 2 does not confirm it. Equilibria of these type may also be the Pareto-efficient equilibria. The purpose in the current paper is not derive all separating equilibria, but merely to show that among those equilibria of the same type as those in games R and games BS, the Pareto inferior ones do not survive standard refinements, while the Pareto superior ones do.
} 
(ii) Player i's strategy supports a strict pure-strategy separating Nash equilibrium for $z^{*}=2$ and a weak pure-strategy separating Nash equilibrium for $z^{*}=3$, but does not support any separating Nash equilibria for $z^{*}>3$.

Proof:

See Appendix.

The intuition for this result is the following. Suppose that Rowena catches Colin playing false, and saying 'OK' even though Rowena did not say anything (false acknowledgement). Rowena now punishes Colin by staying at home, even though there is a good movie on at the cinema. Suppose additionally that Rowena then does not confirm receipt of Colin's 'OK' message. When not receiving a confirmation of receipt of his ' $O K$ ' message, Colin now receives a strong cue that he was caught playing false, and that Rowena is going to stay home. When on the contrary receiving a confirmation of receipt of his ' $\mathrm{OK}$ ' message, Colin knows that his false acknowledgement went undetected, and that he can still meet Rowena at the cinema. Rowena's punishment is not effective, because she is as it were warning Colin of an impending punishment, thus taking much of its power away.

Note that the issue of how to respond to detected false acknowledgements does not arise for equilibria where at most one or two messages are sent. For this reason, these equilibria continue to exist when players do not reply to detected false acknowledgements. The equilibrium where at most two messages are sent is weak, however, because it involves a weak best response by player 1 to the undetected event of observing a false acknowledgement.

As we now go on to show, if player $i$ when detecting a false acknowledgement adopts exactly the same signaling strategy as he or she would have had he or she not detected the acknowledgement to be false, but then still plays $A$, then separating equilibria replicating those in games R and BS exist in game EMG*.

PROPOSITION 5. For sufficiently small $c$ and $d$, for $z^{*}=2$ a strict pure-strategy separating Nash equilibrium exists where, and for each $z^{*} \in[3,4, \ldots, z]$ a weak purestrategy separating Nash equilibrium exists where:

(i) $L_{i}^{* 0}$ such that $\lambda \underset{i}{* z^{*}}=L_{i}^{z^{*}}=\lambda \underset{i}{* z}, 0 \leq\left|\lambda \underset{i}{* z^{*}}-\lambda{ }_{j}^{* z^{*}}\right| \leq 1$;

(ii) $s_{i}^{* t}\left(\left(r_{i}^{t-1}=1\right\}\right)=1$ and $s_{i}^{* t}\left(\left\{r_{i}^{t-1}=0\right\}\right)=0$ for all $t=\left\{(i+1),(i+3), \ldots, z_{i}^{*}\right\}, s_{i}^{* t}=0$

for all $t>z_{i}^{*}$;

(iii) $e_{i}^{*^{z+1}}\left(\left\{\sigma_{i}^{z^{*}}=S_{i}^{z^{*}}\right\} \wedge\left\{\rho_{i}^{z^{*}}=R_{i}^{z^{*}}\right\}\right)=B, e_{i}^{* z+1}\left(\left\{\sigma_{i}^{z^{*}}<S_{i}^{z^{*}}\right\} \vee\left\{\rho_{i}^{z^{*}}<R_{i}^{z^{*}}\right\}\right)=A$, with $i \in\{1,2\}$.

Proof:

See Appendix.

Intuitively, if Rowena wants to stop Colin from saying 'OK' even though he did not hear what Rowena was saying, Rowena should not only not show up at the cinema, but should also still confirm receipt of Colin's false acknowledgement. As Colin now receives a confirmation of receipt both when sending a true acknowledgement and when sending a false one, Colin no longer receives information as to whether his false acknowledgement was detected, and faces the possible cost of ending up at the cinema alone.

Yet, while the separating equilibria described in Proposition 5 for game EMG* replicate those of games $\mathrm{R}$ and $\mathrm{BS}$, the difference is that the Pareto inefficient 
equilibria in game EMG* do not survive standard refinements of the Nash equilibrium. In particular:

PROPOSITION 6. In game EMG*, only pure-strategy separating Nash equilibria with $z^{*} \leq 5$ are perfect Bayesian equilibria.

Proof:

See Appendix.

The intuition for Proposition 6 is simple. Suppose that Rowena has observed that there is a good movie on at the cinema, but that the fact that she has not received a confirmation from Colin causes her not to send any further messages. Still, out of the blue, at some later stage, she receives a confirmation of receipt from Colin. Rowena is now supposed to stay at home, but to still reply to this message. However, since there is no good movie on at home, Rowena obtains payoff 0 whatever Colin's action. Therefore, she does not have any incentive to reply to a false acknowledgement. It is easy to check that a situation where Rowena has observed that there is a good movie on at the cinema, and receives a false acknowledgement to which she should reply only occurs for separating equilibria with $z^{*} \geq 6$.

Consider, however, the Nash equilibrium in Proposition 5 where Rowena sends a confirmation of Colin's confirmation of her invitation $\left(z^{*}=4\right)$. Let Rowena observe that there are no good movies on at the cinema, let her not send an invitation, but let her still get a confirmation of receipt. In this case, it does make sense for Rowena to confirm receipt of this false acknowledgement, and then to stay at home. The reason is that Rowena may believe that she has encountered a freak uninformed player who also sends a message when not receiving an invitation, and only stays at home when receiving a confirmation of this message.

Similarly, consider the Nash equilibrium in Proposition 5 where Rowena sends an invitation to Colin, followed by a confirmation by Colin, where Rowena again confirms receipt of this confirmation, and where Colin finally confirms receipt of the confirmation of the confirmation $\left(z^{*}=5\right)$. Let Colin now not receive an invitation, not confirm receipt of any invitation, but still receive a false acknowledgement from Rowena. It makes sense now for Colin to acknowledge receipt of this false acknowledgement, and then to still stay at home. This is because Colin could conclude that he has encountered a freak informed player who sends a false acknowledgement when having observed that there is no good movie on at the cinema, and only stays at home when receiving a confirmation of this message.

Still, the equilibria with $z^{*}=4$ and $z^{*}=5$ do not survive another standard refinement of the Nash equilibrium:

PROPOSITION 7. In game EMG*, only pure-strategy separating Nash equilibria with $z^{*} \leq 3$ (= Pareto-efficient equilibria) meet the intuitive criterion (Cho and Kreps, 1987).

Proof: See Appendix.

The following problem exists with separating equilibria where $z^{*}=4$ or $z^{*}=5$. Suppose that Colin does not receive an invitation, does not confirm receipt of any invitation, but still receives a false acknowledgement of his supposed confirmation. Then the equilibrium described in Proposition 5 calls for Colin to stay at home. However, this requires Colin to believe that Rowena is planning to stay home, even 
though she invested in the cost of sending a message. But Rowena has no reason to send a message if she is planning to stay home. If she observed that there only is a good movie on television and not at the cinema, she could have induced Colin to stay home without sending a message; if she observed that the there is a good movie on at the cinema, she has no reason to send a message, unless she hopes that this increases the probability that Colin shows up at the cinema. This argument in fact applies to any equilibrium where detected false acknowledgements are punished by playing $A$. A rational player detecting a false acknowledgement from another rational player should reason that this player saw an opportunity to benefit from coordinated action, and that this player expects to achieve coordinated action. Rather than as an act of cheating, a false acknowledgement should be interpreted as an attempt to still achieve coordinated action.

The reason why the equilibrium where Rowena simply sends an invitation to Colin $\left(z^{*}=2\right)$ survives refinements is that the issue of false acknowledgements is not relevant then, as acknowledgements are not sent in the first place. The reason why the equilibrium where Rowena sends an invitation followed by a confirmation by Colin $\left(z^{*}=3\right)$ survives refinements is that Colin prefers not send a false acknowledgement whatever Rowena's response to it.

\section{ELECTRONIC MAIL GAME WITH OUT-OF-EQUILIBRIUM MESSAGES}

Section 5 has shown that, if players use messages that are not proofs of receipt, then only Pareto efficient equilibria survive standard refinements of the Nash equilibrium. As an argument in favor of Pareto-efficient equilibria, this is incomplete, however. The analysis in Section 5 is based on the assumption that players can only use a single type of messages, which are not proofs of receipt. More realistically, players can choose from a set of messages, including messages that take the form of proofs of receipt. If players then happen to coordinate on using proofs of receipt, then Pareto inefficient equilibria would still seem to exist. Fortunately, under the assumption that paying attention at a certain stage means that one detects all messages received at this stage, unused messages that do not take the form of proofs of receipt now play a similar role in destabilizing Pareto inefficient equilibria as do false acknowledgements in game EMG*. In particular, by an analogous argument as in Proposition 7, separating equilibria with $z^{*}>3$ do not meet the intuitive criterion (Cho and Kreps, 1987). Clearly, Rowena only has an incentive to deviate from the equilibrium and send a costly out-of-equilibrium message if she has observed that there is a good movie on a the cinema, and if she intends to go (expecting that Colin is also likely to go to the cinema when receiving the message). Similarly, Colin, once he has received at least one message from Rowena (and thereby finds out that there is a good movie on at the cinema), only has an incentive to send a costly out-of-equilibrium message if he intends to go to the cinema (expecting that Rowena is also likely to go to the cinema when receiving the message).

Intuitively, when player $i$ receives a message in spite of the fact that the message exchange was interrupted, then player $i$ should not interpret this as a signal that player $j$ does not want to cooperate, but on the contrary as a signal that player $j$ still wants to cooperate, in spite of the interrupted message exchange. Clark (1996, pp.284-285) refers to such a process as repair (of the interrupted message exchange), and to such an out-of-equilibrium message as a request for repair. 
A formal proof of this intuition requires modeling a variant of game BS, where on top of proofs of receipt, players can at each relevant stage send an additional message that is not a proof of receipt. This exercise is not undertaken, as it leads to a proposition and a proof very similar to Proposition 7 and its proof.

\section{EVOLUTIONARY STABILITY OF SEPARATING EQUILIBRIA IN THE EMG}

Let us now look at the evolutionary stability of the equilibria derived for the several variants of the EMG treated in this paper. The underlying thought is that the EMG is either symmetrized into a so-called asymmetric contest (Selten, 1980), such that we have a single population of players to whom the role of player 1 or player 2 is attributed. Alternatively, we have an asymmetric population game, with a population of type 1 players and a population of type 2 players.

An immediate result is that all Nash equilibria described in Propositions 2 and 3 are evolutionary stable strategies (ESS; see Maynard Smith and Price (1979) and Selten (1980) for this concept in the case of one population, and see e.g. Swinkels (1992) for the case of multiple populations), as they are all strict Nash equilibria (see Selten, 1980 for this in result in the case of an asymmetric contest; see e.g. Swinkels (1992) for this result in the case of an asymmetric population game). We only have strict separating Nash equilibria in these games because, even if player $i$ deviates from the equilibrium path, it is impossible for player $i$ to produce an observed history for player $j$ that does not already occur with positive probability in equilibrium. We therefore do not have the problem that responses to unobserved histories are necessarily weak best responses. A first reason for this is that messages in games $\mathrm{R}$ and BS are assumed to take the form of proofs of receipt. It is not possible for player $i$ to send an off-the-equilibrium-path false acknowledgement in this case. A second reason is the fact that players do not pay attention at stages where messages are not sent with positive probability in equilibrium, and this either by definition (game R), or because the players' mutual expectations make them do so (game BS).

In game $\mathrm{EMG}^{*}$, the fact that players do not pay attention at stages where messages are not sent with positive probability in equilibrium, again assures that the separating equilibrium with $z^{*}=2$ described in Proposition 5 is a strict Nash equilibrium, and therefore an ESS. Separating equilibria with $z^{*} \geq 3$, however, are weak equilibria; given that messages no longer take the form of proofs of receipt, it is possible for player $i$ to send a false acknowledgement, and to produce an observed history for player $j$ that in equilibrium should be observed with zero probability. The response of player $j$ to such an off-the-equilibrium-path observed history is necessarily a weak best response, and therefore the separating Nash equilibria described in Proposition 5 with $z^{*} \geq 3$ for EMG* are not ESS.

Still, the equilibrium with $z^{*}=3$ described in Proposition 5 for $\mathrm{EMG}^{*}$ can be considered to be evolutionary stable by the fact that it is part of a so-called strict equilibrium set (Balkenborg 1993; Balkenborg and Schlag 2006). A set of Nash equilibria is called a strict equilibrium set if each equilibrium in the set has the following property: if a player $i$ deviates from a Nash equilibrium in the set by using any alternative best reply to player $j$ 's equilibrium strategy, then this only leads to an other Nash equilibrium in the set being played. The separating equilibrium with $z^{*}=3$ described in Proposition 5 is thus part of a strict equilibrium set if every alternative best response of player 1 to a detected false acknowledgement still induces player 2 not to send a false acknowledgement, so that we have a separating Nash 
equilibrium for each alternative best response by player 1 to an undetected false acknowledgement. As shown in the proof to Proposition 8 below, this indeed turns out to be the case for the equilibrium with $z^{*}=3$. Intuitively, when player 2 does not receive a message, then this may occur in spite of the fact that state $b$ occurs, and may be caused by the fact that player 1's message got lost. However, it is more likely that state $a$ occurs, in which case player 2 can obtain benefit $M$ by not sending any message. It follows that player 2 should not send any message.

The fact that the Nash equilibrium with $z^{*}=3$ is part of a strict equilibrium set in turn implies that this equilibrium is part of a so-called evolutionary stable set (ES set; see Thomas (1985) for ES sets in the case of the one-population approach; see Balkenborg and Schlag (1998) for ES sets in the case of a multi-population approach). An ES set is a set of strategies such that all strategies not belonging to the set are driven out by strategies belonging to the set. The result that all strict equilibrium sets are ES sets is due to Balkenborg and Schlag (1995) for the one-population approach, and to Balkenborg and Schlag (1998) for the multi-population approach.

In the case of equilibria with $z^{*}>3$, however, it occurs that the player $i$ who considers sending a false acknowledgement already knows that state $b$ occurs. As long as the other player employs the alternative weak best response of playing $B$ when detecting a false acknowledgement, we no longer have an equilibrium. It follows that these equilibria are not part of a strict equilibrium set, and by extension not part of ES sets. These results are summarized in Proposition 8.

\section{PROPOSITION 8.}

(i) The separating Nash equilibria described in Propositions 1 to 3 for games $R$ and $B S$, as well as the separating Nash equilibrium with $z^{*}=2$ in game $E M G^{*}$, are all ESS.

(ii) The separating Nash equilibrium with $z^{*}=3$ described in Proposition 5 for game $E M G^{*}$ is part of an ES set.

(iii)The separating Nash equilibria of game EMG* with $z^{*}>3$ are not ESS, and are not part of ES sets.

Proof:

See Appendix.

\section{CONCLUSION}

In the example in the introduction to this paper of two game theorists trying to go on a date, for them to meet each other at the cinema most of the time, the players should have common knowledge about each other's conjectures (Aumann and Brandenburger, 1995). But this standard type of interactive knowledge may be only one of two types of interactive knowledge that is being used in this game. Given that Rowena's message that she wants to go on a date with Colin sometimes gets lost, the players may want to achieve some degree of interactive knowledge about the fact that Rowena wants to take Colin on a date. By confirming Rowena's invitation, Colin signals to the Rowena that he knows that she wants to take him on a date. By acknowledging receipt of Colin's confirmation, Colin signals to Rowena that he knows that she knows that he wants to take her on a date. Et cetera. In linguistics, such a process is referred to as grounding (Clark and Schaefer, 1987).

In Clark and Schaefer's model of grounding (1987), in principle the grounding process could never stop. Apparently, with every new acknowledgement sent, 
Rowena and Colin achieve a higher degree of interactive knowledge about the fact that she wants to take him on a date. Yet, as shown by Rubinstein (1989), the players are in fact best off when at most a single acknowledgement is sent. ${ }^{5}$ Still, Binmore and Samuelson (2001) argue that the players' mutual expectations can lock them into requiring an inefficiently large number of confirmations and confirmations of confirmations from each other. Our analysis shows that this result hinges on the assumption that a message can only be acknowledged if it was actually received. And it is true that, in everyday conversation, people sometimes prove that they received a message, e.g. by paraphrasing that message (Clark, 1996, p.228).

Yet, people also use general-purpose acknowledgements such as 'I see' or 'uh huh', which do not provide any evidence that a message was actually received (ibid.). Our analysis shows that, if players use such general-purpose acknowledgements, then at most one acknowledgement will be sent (Section 5). Moreover, grounding processes also involve requests for repair, where an interlocutor signals that he has not understood a preceding signal (Clark, 1996, pp.284-285). As shown in Section 6, requests for repair cause the use of a long sequence of confirmations and confirmations of confirmations to be unstable even for the case where players are currently only communicating by means of proofs of receipt.

Binmore and Samuelson's (2001) argue that the existence of Pareto inefficient separating equilibria where players require a large number of confirmations, and confirmations of confirmations from one another, explains why people sometimes get locked into embarrassingly long conversations to achieve coordinated action. Yet, while the connections between grounding and the concept of common knowledge ("I know that you know that I know that ...") naturally leads one to consider positive acknowledgements, grounding may not exclusively take the form of positive acknowledgements. This is in particular the case for our modified electronic mail game, where each player in turn gets the opportunity of sending a single message, in whatever circumstances he chooses.

Consider the case where Colin tells Rowena that he wants to take her on a date, after which Rowena confirms Colin's invitation. Instead of only sending a message when receiving the confirmation (positive acknowledgement), Colin may instead send a message when not receiving a confirmation (negative acknowledgement), and this in order to still try and obtain a confirmation. In fact, Colin could repeat this process for a number of times, before giving up, and deciding to stay at home.

Also, consider the case where Colin sends a single message. If Rowena does not receive the message, and considers it relatively likely that Colin wants to take here to see a movie, and considers it relatively likely that messages get lost, she may send a negative acknowledgement to Colin, upon which Colin repeats his invitation. Again, Rowena could send a few negative acknowledgements, until she is satisfied that there is no good movie on. In the unlikely event of several misunderstandings, the grounding process may therefore be long. The circumstances under which such equilibria involving negative acknowledgements exist are derived in De Jaegher (2005).

\footnotetext{
${ }^{5}$ The solution to this paradox is to use the notion of common $p$-belief (Monderer and Samet, 1989). An event is common $p$-belief if everyone believes it with probability at least $p$; everyone believes with probability at least $p$ that everyone believes it with probability at least $p$; and so on. It can be checked now that, whether a total of one or more messages is sent in the electronic mail game, it remains common $p$-belief that the state $b$ occurs.
} 


\section{APPENDIX}

\section{Further notation:}

$\tilde{\pi}_{i}^{t}\left(F \mid h_{i}^{t}\right)$ denotes players $i$ 's beliefs at stage $t$ that event $F$ occurs after having observed history $h_{i}^{t}$ (where we sometimes summarize the observed history by the occurrence of one or more events). Denote by $E U_{i}^{t}\left(x_{i}^{t} \mid h_{i}^{t}\right)$ player $i$ 's expected utility at stage $t$ of adopting strategy $x_{i}^{t}$ after having observed history $h_{i}^{t}$.

Player $i$ 's observed history up to stage $(t-1)$ is denoted $h_{i}^{t}=\left(r_{i}^{i}, s_{i}^{i+1}, r_{i}^{i+2}, \ldots, r_{i}^{t-1}\right)$. Players' strategies can be denoted as a function of such an observed history, e.g. function $s_{1}^{t}=\left(\left(r_{1}^{1}, s_{1}^{2}, r_{1}^{3}, \ldots, r_{1}^{t-1}\right)\right)$ relates $i$ 's signaling decision to the observed history up to $t$. $s_{1}^{t}=((\ldots, 1))$ is the function denoting $i$ 's signaling decision whenever $i$ has received the message at $(t-1)$.

\section{Proof of Proposition 1:}

Steps 1 and 2 show that, in any pure-strategy separating equilibrium of game $\mathrm{R}$, $e_{i}^{* z+1}\left(\rho_{i}^{z}<R_{i}^{z}\right)=A$, Step 3 shows that $e_{i}^{*_{i}^{z+1}}\left(\rho_{i}^{z}=R_{i}^{z}\right)=B$.

Step 1. Note that $\tilde{\pi}_{2}^{z+1}\left(\left\{r_{1}^{1}=0\right\} \mid\left\{r_{2}^{2}=0\right\}\right)=\frac{(1-p)}{(1-p)+p(1-\psi)}$. Given that $e_{1}^{* z+1}\left(\left\{r_{1}^{1}=0\right\}\right)=A$, it follows that $E U_{2}^{z+1}\left(e_{2}^{z+1}=A \mid\left\{r_{2}^{2}=0\right\}\right)=\frac{(1-p) M}{(1-p)+p(1-\psi)}$. $E U_{2}^{z+1}\left(e_{2}^{z+1}=B \mid\left\{r_{2}^{2}=0\right\}\right)$ is no higher than $\frac{p(1-\psi) M-(1-p) L}{(1-p)+p(1-\psi)}$. It follows that $e_{2}^{* z+1}\left(\left\{r_{2}^{2}=0\right\}\right)=A$, as by (1) and (2), the former expected utility is smaller than the latter.

Step 2. Let $h_{1}^{z+1}=\left(r_{1}^{1}, s_{1}^{2}, r_{1}^{3}, \ldots\right)=(1,1,0, \ldots)$ (where we suppress the attention paying decisions when denoting observed histories). Then $\tilde{\pi}_{1}^{z+1}\left(\left\{r_{2}^{2}=0\right\} \mid h_{1}^{z+1}\right)=\frac{(1-\psi)}{(1-\psi)+\psi(1-\psi)}=\frac{1}{1+\psi}$, and $E U_{1}^{z+1}\left(e_{1}^{z+1}=A \mid h_{1}^{z+1}\right)=0$. As by Step $1 e_{2}^{*^{z+1}}\left(\left\{r_{2}^{2}=0\right\}\right)=A, E U_{1}^{z+1}\left(e_{1}^{z+1}=B \mid h_{1}^{z+1}\right)$ is no higher than $\frac{\psi M-L}{1+\psi}$. It follows that $e_{1}^{* z+1}\left(h_{1}^{z+1}\right)=A$, as by (2), $\frac{\psi M-L}{1+\psi}<0$. Using this fact, by an identical argument, one can now show that $e_{2}^{*^{z+1}}((1,1,0, \ldots))=A$, which again can be used to show that $e_{1}^{*^{z+1}}((1,1,1,0, \ldots))=A$, etc. In general, $e_{i}^{*_{i}^{z+1}}\left(\left\{\rho_{i}^{z}<R_{i}^{z}\right\}\right)=A$.

Step 3. By Steps 1 and 2, a pure-strategy separating Nash equilibrium only can exist when it is the case that $e_{i}^{* z+1}\left(\left\{\rho_{i}^{z}=R_{i}^{z}\right\}\right)=B$. To show that such an equilibrium indeed exists, first, consider the case where $z_{i}=(z-1)$. Then $\tilde{\pi}_{i}^{z+1}\left(e_{j}^{z+1}=B \mid\left\{\rho_{i}^{z}=R_{i}^{z}\right\}\right)=1$, and $e_{i}^{z+1}\left(\left\{\rho_{i}^{z}=R_{i}^{z}\right\}\right)=B$ is indeed a best response for player $i$. Second, consider the 
case $z_{i}=z$. In this case, $\tilde{\pi}_{i}^{z+1}\left(e_{j}^{z+1}=B \mid\left\{\rho_{i}^{z}=R_{i}^{z}\right\}\right)=\psi$, and $e_{i}^{z+1}\left(\left\{\rho_{i}^{z}=R_{i}^{z}\right\}\right)=B$ is a best response by (3).

Proof of Proposition 2:

Steps 1 and 2 show part (i) of Proposition 2; Step 1 shows that if player $i$ in any purestrategy equilibrium pays attention at a certain stage, he or she will also pay attention at all preceding stages where he or she could have paid attention. Step 2 shows that if player $i$ pays attention at a certain stage, player $j$ (where $j \neq i$ ) will pay attention at all preceding stages where he or she could have paid attention. Step 3 shows part (ii). Step 4 shows part (iii).

Step 1. In game BS, by definition $l_{i}^{t}=0 \Rightarrow s_{i}^{t+1}=0$. The fact that $s_{i}^{t+1}=0$ again implies that $\forall \tau \geq(t+1): s_{j}^{\tau}=0, r_{i}^{\tau}=0$, where $j \neq i$. It follows that if $l^{* t}{ }_{i}^{t}=0$, then $\forall \tau>t: l_{i}^{* \tau}=0$.

Step 2. In game BS, by definition, $l_{i}^{t}=0 \Rightarrow \forall \tau>t: s_{i}^{\tau}=0$. But if player $i$ adopts a strategy such that $\forall \tau>t: s_{i}^{\tau}=0$, then it is a best response for player $j$ (where $j \neq i$ ) to adopt a strategy where $\forall \tau>t: l_{j}^{\tau}=0$.

By Steps 1 and 2, in any pure-strategy separating equilibrium of game BS, the players only pay attention at a range of contiguous stages.

Step 3. If player $i$ puts $l_{i}^{t}=0$, then it is a best response for player $j$ (where $j \neq i$ ) to put $s_{j}^{t}=0$. If player $j$ puts $s_{j}^{t}=0$, it is a best response for player $i$ to put $l_{i}^{t}=0$. It follows that, in any pure-strategy separating equilibrium of game BS, if $l{ }^{* t}=1$, then $S^{* t}\left(\left\{r_{j}^{t-1}=1\right\}\right)=1$.

Steps 1 to 3 together show that, in any pure-strategy separating equilibrium of game BS, player $i$ sends a proof of receipt for each stage at which player $j$ pays attention.

Step 4: Part (iii) of Proposition 2 follows by an argument identical to the one made for Proposition 1.

\section{Proof of Proposition 3:}

Let player $j$ follow the strategy of the candidate equilibrium corresponding to a given $z^{*}$. We show that it is a best response then for player $i$ (where $i \neq j$ ) to follow the corresponding candidate equilibrium strategy.

Steps 1 and 2 show that, when player $j$ follows the candidate equilibrium, it is either a best response for player $i$ to pay attention at least at all stages preceding the stages where player $j$ requires a message in order to play $B$, or it is a best response for $i$ not to pay attention at all. Step 3 shows that, for sufficiently small attention paying and signaling costs, the former is a best response. Step 4 shows that, if player $j$ is the last who can send a message in equilibrium, then it is a best response for player $i$ to pay attention at the stage where this message is sent.

Step 1. Let $\lambda_{i}^{z^{*}}<S_{i}^{z^{*}}$. Then by definition, $\sigma_{i}^{z^{*}}<S_{i}^{z^{*}}$, from which it follows that $\rho_{j}^{z^{*}}<R_{j}^{z^{*}}$. As $e_{j}^{*^{z+1}}\left(\left\{\rho_{j}^{z^{*}}<R_{j}^{z^{*}}\right\}\right)=A$, it follows that $e_{i}^{z+1}\left(\left\{\lambda_{i}^{z^{*}}<S_{i}^{z^{*}}\right\}\right)=A$. Given that $c>0$, it follows that it is never a best response for player $i$ to put $0<\lambda_{i}^{z^{*}}<S_{i}^{z^{*}}$. Thus, in one candidate best response, it is the case that $\lambda_{i}^{z^{*}}=0, \sigma_{i}^{z^{*}}=0, e_{i}^{z+1}=A$. 
Step 2. Let $\lambda_{i}^{z^{*}}=S_{i}^{z^{*}}, S_{i}^{z^{*}} \leq \lambda_{i}^{z^{*}} \leq R_{i}^{z^{*}}$.

Case 1. Let $\sigma_{i}^{z^{*}}=S_{i}^{z^{*}}$. Consider first the case where $\lambda_{i}^{z^{*}}=R_{i}^{z^{*}}$. Then by Proposition 1, $e_{i}^{z+1}\left(\left\{\sigma_{i}^{z^{*}}=S_{i}^{z^{*}}\right\} \wedge\left\{\rho_{i}^{z^{*}}=R_{i}^{z^{*}}\right\}\right)=B$. Second, consider the case not covered by Proposition 1 where $z_{j}^{*}=z^{*} \quad$ and $\quad \lambda_{i}^{z^{*}}=S_{i}^{z^{*}}=\left[R_{i}^{z^{*}}-1\right]$. Then $\tilde{\pi}_{i}^{z+1}\left(e_{j}^{z+1}=B \mid\left\{\sigma_{i}^{z^{*}}=S_{i}^{z^{*}}\right\} \wedge\left\{\rho_{i}^{z^{*}}=\left[R_{i}^{z^{*}}-1\right]\right\}\right)=\psi, \quad$ and $\quad$ by $e_{i}^{z}\left(\left\{\sigma_{i}^{z^{*}}=S_{i}^{z^{*}}\right\} \wedge\left\{\rho_{i}^{z^{*}}=\left[R_{i}^{z^{*}}-1\right]\right\}\right)=B$.

Case 2. Let $h_{i}^{z+1}$ be such that $\sigma_{i}^{\tau}=S_{i}^{\tau}=\sigma_{i}^{z^{*}}$, with $\tau<z_{i}^{*}$. First, $h_{i}^{z+1}$ may occur because $\rho_{i}^{\tau-1}=R_{i}^{\tau-1}=\rho_{i}^{z^{*}}$. By Proposition 1 , it is then the case that $e_{i}^{z+1}\left(h_{i}^{z+1}\right)=A$. Second, not covered in Proposition $1, h_{i}^{z+1}$ may occur in spite of the fact that $\rho_{i}^{\tau+1}=\rho_{i}^{z^{*}}=R_{i}^{\tau+1}$. As $\sigma_{i}^{z^{*}}<S_{i}^{z^{*}}$, we have $\rho_{j}^{z^{*}}<R_{j}^{z^{*}}$. As $e_{j}^{*_{j+1}}\left(\left\{\rho_{j}^{z^{*}}<R_{j}^{z^{*}}\right\}\right)=A$, it is met that $e_{i}^{z+1}\left(h_{i}^{z+1}\right)=A$.

It follows that an alternative candidate best response to the one derived in Step 1 has $\lambda_{i}^{z^{*}}$ such that $S_{i}^{z^{*}} \leq \lambda_{i}^{z^{*}} \leq R_{i}^{z^{*}} ; \quad s_{i}^{t}\left(L_{i}^{0}, \ldots, 1\right)=1, \quad s_{i}^{t}\left(L_{i}^{0}, \ldots, 0\right)=0 \quad$ for all $t \in\left\{(i+1),(i+3), \ldots, z_{i}\right\} ; \quad e_{i}^{z+1}\left(\left\{\sigma_{i}^{z^{*}}=S_{i}^{z^{*}}\right\} \wedge\left\{\rho_{i}^{z^{*}}=\lambda_{i}^{z^{*}}\right\}\right)=B, \quad$ and $e_{i}^{z+1}\left(\left\{\sigma_{i}^{z^{*}}<S_{i}^{z^{*}}\right\} \vee\left\{\rho_{i}^{z^{*}}<\lambda_{i}^{z^{*}}\right\}\right)=A$.

Step 3. For sufficiently small $c$ and $d$, player $i$ prefers the responses derived in Step 2 to the one derived in Step 1, as only the response in Step 2 yields a nonzero expected payoff.

Step 4. Let $z_{j}^{*}=z^{*}$. We have shown in Step 3 that player $i$ prefers either putting $\lambda_{i}^{z^{*}}=S_{i}^{z^{*}}$ or putting $\lambda_{i}^{z^{*}}=R_{i}^{z^{*}}$ to putting $\lambda_{i}^{z^{*}}=0$. It remains to be shown that player $i$ prefers putting $\lambda_{i}^{z^{*}}=R_{i}^{z^{*}}$ to putting $\lambda_{i}^{z^{*}}=S_{i}^{z^{*}}$. Note in this respect that player $i$ 's attention paying and signaling decisions are identical up to stage $\left(z^{*}-1\right)$ for these two strategies. In order to compare the expected payoff of these two strategies, it therefore suffices to compare $E U_{i}^{z^{*-1}}\left(\rho_{i}^{z^{*}-1}=\left[S_{i}^{z^{*}}-1\right] \lambda_{i}^{z^{*}}=R_{i}^{z^{*}}\right)$ and $E U_{i}^{z^{*}-1}\left(\rho_{i}^{z^{*}-1}=\left[S_{i}^{z^{*}}-1\right] \mid \lambda_{i}^{z^{*}}=S_{i}^{z^{*}}\right)$. Net of attention paying and signaling costs, the former equals $\psi^{2} M$, the latter equals $[\psi M-(1-\psi) L]$, so that for sufficiently small $c$ and $d$, player $i$ will choose $\lambda_{i}^{z^{*}}=R_{i}^{z^{*}}$.

\section{Proof of Proposition 4:}

Assume that player $j$ follows the candidate equilibrium strategy for a given $z^{*}$; our approach again is to check whether it is a best response for player $i$ (where $i \neq j$ ) to follow the candidate equilibrium strategy as well.

Step 1. Propositions 1 to 3 can be used to show that, in the set of strategy profiles with player $i$ 's action sets restricted in such a way that $s_{i}^{t}\left(\left\{r_{i}^{t-1}=0\right\}\right)=0$ and $s_{i}^{t}\left(\left\{l_{i}^{t-1}=0\right\}\right)=0$ for all $t=\left\{(i+1),(i+3), \ldots, z_{i}^{*}\right\}$, the candidate equilibrium strategy is a best response for player $i$. However, in game $\mathrm{EMG}^{*}$, the action sets are not restricted. It follows that it remains to be checked whether $s_{i}^{t}\left(\left\{r_{i}^{t-1}=0\right\}\right)=0$ is a best response, and whether it is not a best response to put $s_{i}^{t}\left(\left\{l_{i}^{t-1}=0\right\}\right)=1$. However, if a player $i$ 
who has put $l_{i}^{t-1}=1$ prefers to put $s_{i}^{t}\left(\left\{r_{i}^{t-1}=0\right\}\right)=0$ and $s_{i}^{t}\left(\left\{r_{i}^{t-1}=1\right\}\right)=1$, then for sufficiently small $c$ this player $i$ will put $l_{i}^{t-1}=1$. This is why we further assume that $\lambda_{i}^{z^{*}}=\lambda_{i}^{z}=L_{i}^{z^{*}}$.

Step 2 shows that, if player 2 is following any candidate equilibrium, then $s_{1}^{2}\left(\left\{r_{1}^{1}=0\right\}\right)=0$. Step 3 shows that, if player 1 is following the candidate equilibrium with $z^{*}=3$, then $s_{2}^{3}\left(\left\{r_{2}^{2}=0\right\}\right)=0$. Step 4 shows that, in any equilibrium with $z^{*}>3$ where $z_{i}^{*}=z^{*}$, if player $j$ (where $j \neq i$ ) follows the candidate equilibrium, then $s_{i}^{z^{*}}\left(\left\{\sigma_{i}^{z^{*-2}}=\rho_{i}^{z^{*-3}}=S_{i}^{z^{*}-2}\right\} \wedge\left\{r_{i}^{z^{*}-1}=0\right\}\right)=0$. Step 5, however, shows that, if player 1 is following a candidate equilibrium with $z^{*}>3$, then $s_{2}^{3}\left(\left\{r_{2}^{2}=0\right\}\right)=1$. Step 6 finally shows that, in any equilibrium with $z^{*}>4$, the candidate equilibrium strategy does not stop players from sending false acknowledgements at stages $t$ with $3 \leq t \leq\left(z^{*}-1\right)$.

Step 2. Let player 2 follow the strategy of one of the candidate equilibria . Consider player 1's decision whether to send the message at stage 1. Given that $e_{2}^{*^{z+1}}\left(\left\{r_{2}^{2}=0\right\}\right)=A$, it is a best response for player 1 to put $s_{1}^{2}\left(\left\{r_{1}^{1}=0\right\}\right)=0$. It follows as well that the candidate equilibrium with $z^{*}=2$ is an equilibrium.

Step 3. Consider the candidate separating equilibrium with $z^{*}=3$. Assume player 1 follows the candidate equilibrium strategy. Suppose that player 2 follows the strategy $s_{2}^{3}\left(\left\{r_{2}^{2}=0\right\}\right)=1$. Then by Step 1 of Proposition 1 , player 2 prefers to put $e_{2}^{4}\left(\left\{r_{2}^{2}=0\right\} \wedge\left\{s_{2}^{3}=1\right\}\right)=A$. It follows that player 2 prefers to put $s_{2}^{3}\left(\left\{r_{2}^{2}=0\right\}\right)=0$. By Step 1 and Step 2 above, it follows that the candidate equilibrium exists.

Step 4. Consider a candidate equilibrium with $z^{*}>3$ where $z_{i}^{*}=z^{*}$. Let player $j$ (where $j \neq i$ ) follow the candidate equilibrium, and let $h_{i}^{z^{*}}$ be such that $\sigma_{i}^{z^{*}-2}=\rho_{i}^{z^{*}-3}=S_{i}^{z^{*}-2}, r_{i}^{z^{*}-1}=0$. Denote by $P_{i}^{z^{*}}$ player $i$ 's information set in this case. Given that $\sigma_{i}^{z^{*-2}}>1$ when $z^{*}>3$, and given that player $j$ follows the candidate equilibrium strategy, it follows that $\forall h^{z^{*}} \in P_{i}^{z^{*}}:\left\{r_{1}^{1}=0\right\} \notin h^{z^{*}}$, or in other words, player $i$ knows that state $b$ occurs when observing $h_{i}^{z^{*}}$. It is then a best response for player $i$ to put $s_{i}^{z^{*}}\left(h_{i}^{z^{*}}\right)=0$ as it is met that

$$
\frac{\psi[\psi M-(1-\psi) L]-L}{1+\psi}<0,
$$

meaning that player $i$ prefers to play $A$ even after having sent a false acknowledgement at stage $z^{*}$.

Step 5. Consider any candidate separating equilibrium with $z^{*}>3$. Assume that player 1 follows the candidate equilibrium strategy. Assume that player 2 considers either following the candidate equilibrium strategy, or following an alternative strategy identical to the candidate equilibrium strategy with the exception that $s_{2}^{3}\left(\left\{r_{2}^{2}=0\right\}\right)=s_{2}^{3}\left(\left\{r_{2}^{2}=1\right\}\right)=1, e_{2}^{z+1}\left(\left\{r_{2}^{2}=0\right\} \wedge\left\{\rho_{2}^{z^{*}}=\left[R_{2}^{z^{*}}-1\right]\right\} \wedge\left\{\sigma_{2}^{z^{*}}=S_{2}^{z^{*}}\right\}\right)=B . \quad$ Let $r_{2}^{2}=0$. Net of signaling costs, player 2 obtains a higher expected payoff from the alternative strategy than from the candidate equilibrium given that 


$$
\frac{p \psi^{z^{*}-3} w+(1-p) M}{(1-p)+p(1-\psi)}>\frac{(1-p) M}{(1-p)+p(1-\psi)}
$$

where $w=\psi M$ if $z_{2}^{*}=\left(z^{*}-1\right)$ and $w=[\psi M-(1-\psi) L]$ if $z_{2}^{*}=z^{*}$. It follows that for sufficiently small $d$, player 2 prefers to deviate from the candidate equilibrium strategy.

Step 6. Consider any candidate separating equilibrium with $z^{*}>4$. Assume player $j$ follows the candidate equilibrium. Let player $i$ (with $i \neq j$ ) be able to receive the message at stage $\tau$, where $\tau>4$. Assume that player $i$ follows the candidate equilibrium, with the exception of the case where he or she observes $h^{\tau-1}$ such that events $\left\{\rho_{i}^{\tau-4}=R_{i}^{\tau-4}\right\},\left\{\sigma_{i}^{\tau-3}=S_{i}^{\tau-3}\right\}, \quad\left\{r_{i}^{\tau-2}=0\right\}$ occur. Denote by $P_{i}^{\tau-1}$ player $i$ 's information set in this case. Given that $z^{*}>4$ and that player $j$ follows the candidate equilibrium strategy, it follows that $\forall h^{\tau-1} \in P_{i}^{\tau-1}:\left\{r_{1}^{1}=0\right\} \notin h^{\tau-1}$.

Consider the case where player $i$ puts $s_{i}^{\tau-1}\left(h^{\tau-1}\right)=0$. Then $\sigma_{i}^{z^{*}}<S_{i}^{z^{*}}$, meaning that $\rho_{j}^{z^{*}}<R_{j}^{z^{*}}, \underset{j}{*{ }_{j}^{z+1}}\left(\left\{\rho_{j}^{z^{*}}<R_{j}^{z^{*}}\right\}\right)=A$, so that player $i$ puts $a_{i}^{z+1}=A$.

Consider instead the case where player $i$ puts $s_{i}^{\tau-1}\left(h^{\tau-1}\right)=1$. First, if event $\left\{r_{i}^{\tau}=1\right\}$ now occurs, then given that player $j$ follows the candidate equilibrium, observed history $h_{i}^{\tau+1}$ is such that player $i$ is in exactly the same information set as with observed history $h_{i}^{\tau+1}$ such that $\rho_{i}^{\tau}=R_{i}^{\tau}$. Assume that player $i$ adopts the same strategy when observing $h_{i}^{\tau+1}$ as the strategy that he adopts in the candidate equilibrium when observing $h_{i}^{\tau+1}$.

Second, if it is instead the case that $r_{i}^{\tau+1}=0$, assume that player $i$ follows exactly the same strategy as he does in the candidate equilibrium upon observed history $h_{i}^{\prime \prime \tau+1}$ such that events $\left\{\rho_{i}^{\tau-4}=R_{i}^{\tau-4}\right\},\left\{\sigma_{i}^{\tau-3}=S_{i}^{\tau-3}\right\},\left\{r_{i}^{\tau-2}=0\right\},\left\{s_{i}^{\tau-1}=0\right\}$ and $\left\{r_{i}^{\tau}=0\right\}$ occur.

Net of attention paying and signaling costs, player $i$ 's expected payoff from putting $s_{i}^{\tau-1}\left(h^{\tau-1}\right)=1$ (and adopting the assumed strategy) is larger than the expected payoff of putting $s_{i}^{\tau-1}\left(h^{\tau-1}\right)=0$ (and adopting the assumed strategy), as

$$
\frac{\psi^{z^{*}-\tau+2} w}{1+\psi}>0
$$

where $w=\psi M$ if $z_{i}^{*}=\left(z^{*}-1\right)$ and $w=[\psi M-(1-\psi) L]$ if $z_{i}^{*}=z^{*}$. It follows that, for sufficiently small $c$ and $d$, it is a superior response for player $i$ to put $s_{i}^{\tau-1}\left(h^{\tau-1}\right)=1$.

\section{Proof of Proposition 5:}

Assume that player $j$ follows the candidate equilibrium for a given $z *$. We show that it is a best response then for player $i$ (where $i \neq j$ ) to also follow the candidate equilibrium. By Step 1 of Proposition 4, in order to show this, it suffices to show that player $i$, after having decided to put $\lambda_{i}^{z^{*}}=\lambda_{i}^{z}=L_{i}^{z^{*}}$, prefers not to send any false acknowledgements. If we can show this, then it immediately follows that, for sufficiently small $c$, player $i$ will also make the right attention paying decisions. In 
order to show that player $i$ does not send any false acknowledgements, we show that, if player $i$ has sent any number of false acknowledgements, he prefers to play $A$. It follows then immediately that it is a best response for player $i$ not to send any false acknowledgements.

For the purpose of this proof, it is useful to see player $i$ as being able to send a confirmation of the message received from player $j$ at stages $\left\{(5-i),(7-i), \ldots, z_{i}\right\}$, where $K_{i}^{t}$ is the maximum number of confirmations that player $i$ could send up to stage $t$, and $\kappa_{i}^{t}$ is the actual number of confirmations that player $i$ sends up to stage $t$. Similarly, we can see player $i$ as being able to receive the message from player $j$ at stages $\left\{(4-i),(6-i), \ldots, z_{j}\right\}$, where $M_{i}^{t}$ is the maximum number of messages that player $i$ could receive from player $j$ up to stage $t$, and $m_{i}^{t}$ is the actual number of messages that player $i$ receives up to stage $t$.

If $\kappa_{i}^{z^{*}}<K_{i}^{z^{*}}$, then $\rho_{j}^{z^{*}}<R_{j}^{z^{*}}$. As $e_{j}^{*_{j}^{z+1}}\left(\left\{\rho_{j}^{z^{*}}<R_{j}^{z^{*}}\right\}\right)=A$, it follows that $e_{i}^{z+1}\left(\left\{K_{i}^{z^{*}}<K_{i}^{z^{*}}\right\}\right)=A$, whether or not player $i$ sent false acknowledgements. Look now at the case where $\kappa_{i}^{z^{*}}=K_{i}^{z^{*}}$. Step 1 treats the case where player $i$ beliefs with probability 1 that $r_{1}^{1}=1$, Step 2 the case where play $i$ beliefs that $r_{1}^{1}=1$ with a probability smaller than 1 .

Step 1. Consider the case where $i=1$, or where $i=2$ and $r_{2}^{2}=1$. Let $\kappa_{i}^{\tau}=K_{i}^{\tau}$, but let $\mu_{i}^{\tau}=\left[K_{i}^{\tau}-v\right]$ (meaning that player $i$ sent $v$ false acknowledgements, where $\left.1 \leq v \leq K_{i}^{\tau}-(i-1)\right)$. Then player $i$ prefers to put $e_{j}^{z+1}\left(\left\{\kappa_{i}^{\tau}=K_{i}^{\tau}\right\} \wedge\left\{\mu_{i}^{\tau}=\left[K_{i}^{\tau}-v\right]\right\}\right)=A$ as

$$
\left[\frac{\psi}{1+\psi}\right]^{v} w-\left\{1-\left[\frac{\psi}{1+\psi}\right]^{v}\right\} L<0,
$$

where $w=[\psi M-(1-\psi) L]$ if $z_{i}^{*}=z^{*}$ and $w=M$ if $z_{i}^{*}=\left(z^{*}-1\right)$.

Step 2. Consider the case where $h_{2}^{z+1}$ is such that $r_{2}^{2}=0, \sigma_{2}^{z^{*}}=S_{2}^{z^{*}}$. Then the best that player 2 can do by playing $B$ is to obtain $[\rho M-(1-\rho) L]$, which is smaller than the payoff $(1-\rho) M$ from playing $A$.

It follows from Steps 1 and 2 that a player who has sent false acknowledgements prefers to play $A$. But given that this is the case, players prefer not to send false acknowledgements in the first place.

\section{Proof of Proposition 6:}

In the equilibrium with $z^{*}=2$, the problem of detected false acknowledgements does not arise, and in the equilibrium with $z^{*}=3$, by Step 3 of Proposition 4, player 2 prefers not to send a false acknowledgement, whatever player 1's response to it.

Step 1 checks whether the equilibrium with $z^{*}=4$ can be a perfect Bayesian equilibrium. Steps 2 and 3 do the same for the equilibria with $z^{*}=5$ and $z^{*}>5$ respectively.

Step 1. Consider a candidate equilibrium with $z^{*}=4$. Let player 1 put $s_{1}^{4}((0,0,1))=1$ and $e_{1}^{5}((0,0,1,1))=A$ (see Proposition 5), where we suppress players' 
attention paying decisions when denoting observed histories. Such a strategy by player 1 is justified if, upon an observed history $h_{1}^{4}=(0,0,1)$, he believes that he has encountered a freak type of player 2 who adopts strategy $e_{2}^{5}((0,1,0))=B$, $e_{2}^{5}((0,1,1))=A$.

Let player 2 follow $e_{2}^{5}((0,0,1))=A$. Then given (A1) it is a best response for player 1 to follow $s_{1}^{4}((1,1,0))=0$. Player 2 's response is justified if he believes upon an observed history $h_{2}^{5}=(0,0,1)$ that it is likely that player 1 will put $e_{1}^{5}=A$.

Step 2. Consider an equilibrium with $z^{*}=5$. Let player 2 follow $s_{2}^{5}((0,0,1))=0$ and $e_{2}^{6}((0,0,1,0))=A$. Then by Step 6 of Proposition 4 , player 1 will follow $s_{1}^{4}((1,1,0))=1$. It follows that the stated strategy by player 2 cannot support an equilibrium with $z^{*}=5$.

Assume instead that player 2 follows the rule: $s_{2}^{5}((0,0,1))=0$, and $e_{2}^{6}((0,0,1,0))=B$ with positive probability. Then player 1 will be at least as well off when following $s_{1}^{4}((1,1,0))=1$.

It follows that, in order for player 1 to follow $s_{1}^{4}((1,1,0))=0$, it must be the case that player 2 with positive probability follows the strategy $s_{2}^{5}((0,0,1))=1$ combined with $e_{2}^{6}((0,0,1,1))=A$. Such a response by player 2 can be justified by the following beliefs. Player 2 could believe, when observing $h_{2}^{5}=((0,0,1))$, that he is facing a player 1 who observed $h_{1}^{4}=((0,0,0))$, and adopts strategy $s_{1}^{4}((0,0,0))=1, \quad e_{1}^{6}((0,0,0,1,1))=A$, $e_{1}^{6}((0,0,0,1,0))=B$.

Step 3. Consider an equilibrium with $z^{*}>5$. Assume player 1 follows the candidate equilibrium strategy described in Proposition 4 . Let player 2 be able to send the message at stage $(\tau-1)$, where we both consider the case $\tau=z^{*}$, and the case $\tau=\left(z^{*}-1\right)$. Then by Step 6 of Proposition 4, it does not suffice for player 1, upon an observed history $h_{1}^{\tau}=(1, \ldots, 0,0,1)$ to put $s_{1}^{\tau}\left(h_{1}^{\tau}\right)=0, e_{1}^{z+1}=A$ to stop player 2 from always following $s_{2}^{\tau-1}=1$. It follows that, with positive probability, player 1 must put $s_{1}^{\tau}\left(h_{1}^{\tau}\right)=1, e_{1}^{z+1}=A$. However, this is a dominated strategy for player 1 , and there are no beliefs for player 1 that justify such a strategy.

\section{Proof of Proposition 7:}

Consider equilibria with $z^{*} \geq 4$. Let player $i$ detect a false acknowledgement at stage $z^{*}: h_{i}^{z^{*}+1}=\left(\ldots, r_{i}^{z^{*}-2}, s_{i}^{z^{*}-1}, r_{i}^{z^{*}}\right)=(\ldots, 0,0,1)$. If relevant $\left(z^{*} \geq 5\right)$, let $\rho_{i}^{z^{*}-4}=R_{i}^{z^{*}-4}$, $\sigma_{i}^{z^{*}-3}=S_{i}^{z^{*}-3}$, meaning that player $i$ did not detect any other false acknowledgements. Define a player $j$ 's type as a pair $\left(h_{j}^{z^{*}+1}, e_{j}^{z^{*}+1}\right)$ consisting of player $j$ 's observed history $h_{j}^{z^{*+1}}=\left(\ldots, r_{j}^{z^{*-3}}, s_{j}^{z^{*}-2}, 0,1\right)$ (with, if relevant $\sigma_{j}^{z^{*-4}}=S_{j}^{z^{*}-4}$, compatible with player $i$ 's observed history specified above) and of the action $e_{j}^{z^{*}+1}$ that he or she takes. Denote by $T$ the set of all such types.

Whether a Nash separating equilibrium does not meet the intuitive criterion is now checked in the following manner (Cho and Kreps, 1987). Denote by 
$E U_{j}^{* z^{*+1}}((\ldots, 0,0))$ player $j$ 's expected payoff when following the equilibrium path, meaning that player $j$ puts $s_{i}^{z^{*}}=0$ when observing $r_{i}^{z^{*}-1}=0$.

Step 1. Look for set of types $T^{\prime}$ with typical element $\left(h_{j}^{z^{*}+1}, e_{j}^{z^{*}+1}\right)$ meeting

$$
E U_{j}^{* z^{*}+1}((\ldots, 0,0))>\max _{e_{i}^{z^{*+1}}} E U_{j}^{z^{*}+1}\left(h_{j}^{z^{*}+1}, e_{j}^{z^{*}+1}\right)
$$

Thus, the set of types $T^{\prime}$ are then types that will never put $s_{j}^{z^{*}}=1$ and at the same time choose $e_{j}^{z^{*}+1}$. Denote by $T^{\prime \prime}$ the set that meets $T^{\prime \prime}=T \backslash T^{\prime}$. Then a rational player $i$ who observes $h_{i}^{z^{*+1}}=(\ldots, 0,0,1)$ should conclude that player $j$ belongs to the set $T^{\prime \prime}$. Given that player $j$ belongs to the set $T^{\prime \prime}$, player $i$ will then choose his or her best response $e_{i}^{\prime z^{*+1}}$ (where it will turn out that the best response to all the types in $T^{\prime \prime}$ is the same).

Step 2. The equilibrium considered does not meet the intuitive induction criterion if, for a player of type $t^{\prime \prime} \in T^{\prime \prime}$ it is met that

$$
E U_{j}^{* z^{*+1}}((\ldots 0,0))<E U_{j}^{z^{*}+1}\left(t^{\prime \prime}, e_{i}^{\prime \prime z^{*}}\right) .
$$

These two steps are now considered both for the case where $i=1$ and for the case where $i=2$, showing that the corresponding equilibria do not meet the intuitive criterion. LHS and RHS stand for left- and right-hand side respectively.

(i) Consider the case where $i=1,\left\{r_{1}^{1}=1\right\}$. Consider first the types of player 2 with $\rho_{2}^{z^{*}-1}=0, \sigma_{2}^{z^{*}}=S_{2}^{z^{*}}$. After having put $\sigma_{2}^{z^{*}-2}=S_{2}^{z^{*}-2}$, when again following the equilibrium path by putting $s_{2}^{z^{*}}=0$, the LHS of (A5) net of signaling and attention paying costs is $\frac{(1-p) M}{(1-p)+p(1-\psi)[(1-\psi)+\psi(1-\psi)]^{\sigma_{2}^{z^{*}-1}}}$. For the type of player 2 with additionally $e_{2}^{6}=A$, the LHS is equal to $\frac{(1-p) M}{(1-p)+p(1-\psi)[(1-\psi)+\psi(1-\psi)]^{z_{2}^{*}-1}}$, but includes the cost of sending an extra message. For the type with additionally $e_{2}^{6}=B$, the RHS is equal to $\frac{p(1-\psi)[(1-\psi)+\psi(1-\psi)]^{\sigma_{2}^{*}-1}[\psi M-(1-\psi) L]}{(1-p)+p(1-\psi)[(1-\psi)+\psi(1-\psi)]^{\sigma_{2}^{*}-1}}$, and includes the cost of sending an extra message. Each time, (A5) is met.

Second, consider the types of player 2 with $r_{2}^{t}=1$ for some $\tau \geq 3, r_{2}^{\tau}=0$ for $\tau>t .\left\{r_{1}^{1}=0\right\}$ is not an element of such a player's information set. After having put $\sigma_{2}^{z^{*-2}}=S_{2}^{z^{*-2}}$, when again following the equilibrium path by putting $s_{2}^{z^{*}}=0$, the LHS of (A5) net of signaling and attention paying costs is 0 . For the type with additionally $e_{2}^{z^{*}+1}=A$, the difference between the LHS and the RHS of (A5) is $d$.

It follows that the set $T^{\prime \prime}$ consists of types $t^{\prime \prime}$ characterized by $h_{2}^{z^{*}+1}$ such that, for some $t<\left(z^{*}-1\right)$, it the case that $r_{2}^{t}=1$, and by the action $e_{2}^{6}=B$. It follows that $e^{\prime \prime 6}=B$, and that (A6) is met as the RHS is $(M-d)$ larger than the LHS. 
(ii) Consider the case where $i=2$. First, consider the types of player 1 with $h_{1}^{z^{*}+1}=(0, \ldots)$. Then the RHS of (A5) is equal to $M$, and (A5) is necessarily met for all such $h_{1}^{5}$. Second, consider the type of player 1 with $h_{1}^{5}=(1, \ldots)$ and with $e_{1}^{5}=A$. (A5) is now met, as the LHS is now at least $d$ larger than the RHS. The set $T^{\prime \prime}$ therefore consists of types $t^{\prime \prime}$ characterized by $h_{1}^{z^{*}+1}=(1, \ldots)$ and $e_{1}^{5}=B$. It follows that $e_{2}^{\prime z^{*}+1}=B$, and (A6) is met as the difference between LHS and RHS is at least $(M-d)$.

Proof of Proposition 8:

Proof that the equilibrium described in Proposition 5 with $z^{*}=3$ is part of an ES set (part (ii)):

Let player 1 play $A$ with probability $q_{A}$ when having detected a false acknowledgement. Consider first the case where player 2 plays $A$ after having sent a false acknowledgement. Then player 2 prefers not to send a false acknowledgement as

$$
\frac{(1-\rho) M}{(1-\rho)+\rho(1-\psi)}>\frac{(1-\rho)\left[\psi q_{A}+(1-\psi)\right] M}{(1-\rho)+\rho(1-\psi)}-d
$$

Consider second the case where player 2 plays $B$ after having sent a false acknowledgement. Then player 2 prefers not to send a false acknowledgement as

$$
\left.\frac{(1-\rho) M}{(1-\rho)+\rho(1-\psi)}>\frac{-(1-\rho)\left[\psi q_{A}+(1-\psi)\right] L+\rho(1-\psi)[\psi M-(1-\psi) L]}{(1-\rho)+\rho(1-\psi)}-d, \text { (A } 8\right)
$$

where (A8) follows from the fact that, by (1) and (2), the right-hand side of (A8) is smaller than zero.

\section{References}

Aumann, R.J., Brandenburger, A., 1995. Epistemic conditions for Nash equilibrium. Econometrica 63, 1161-1180.

Balkenborg, D., 1993. Strictness and evolutionary stability. Discussion paper, Center for Rationality and Interactive Decision Making, Hebrew University of Jerusalem.

Balkenborg, D., Schlag, K., 1995. On the interpretation of evolutionary stable sets. Working paper, Department of Economics, University of Bonn.

Balkenborg, D., Schlag, K., 1998. Evolutionary stable sets in population games. Working paper, Department of Economics, University of Bonn.

Balkenborg, D., Schlag, K., 2006. On the evolutionary selection of sets of Nash equilibria. Journal of Economic Theory, in press.

Binmore, K., Samuelson, L., 2001. Coordinated action in the electronic mail game. Games Econ. Behav. 35, 6-30.

Cho, I.-O., Kreps, D.M. 1987. Signaling Games and Stable Equilibrium. Quart. J. Econ. 102, 179-222. 
Christensen, S., 2001. Formation of contracts by email - is it just the same as post? Queensl. Univ. of Technol. Law \& Justice J. 1, 22-38.

Chwe, M. S.-Y., 1995. Strategic reliability of communication networks. working paper, University of Chicago, Department of Economics.

Clark, H.H., 1996. Using Language. Cambridge University Press, Cambridge.

Clark, H.H., Schaefer, E.F., 1987. Collaborating on contributions to conversations. Lang. Cognit. Process. 2, 19-41.

De Jaegher, K. 2005. Game-theoretic grounding. In: Benz, T., Jäger, G., Rooij, R van (Eds.), Games and Pragmatics, Palgrave Macmillan, London.

De Jaegher, K., 2004. Sequential costly signalling. working paper, Vrije Universiteit Brussel, Department of Economics.

Geanakoplos, J., 1992. Common knowledge. J. Econ. Perspect. 6, 53-82.

Gray, J.N., 1978. Notes on database operating systems. In: Bayer, R., Graham, R.M., Seegmuller, G. (Eds.) Operating Systems: an Advanced Course, Springer Verlag, New York, pp. 393-481.

Halpern, J. and Moses, Y., 1984. Knowledge and common knowledge in a distributed environment. Proceedings of the $3^{\text {rd }}$ ACM Symposium on Principles of Distributed Computing, 1984.

Kreps, D.M., Wilson, R., 1982. Sequential equilibria. Econometrica 50, 863-894.

Matsui, A., 1992. Best response dynamics and socially stable strategies. J. Econ. Theory 57, 343-362.

Maynard Smith, J., Price, G.R., 1973. The logic of animal conflict. Nature 246, 15-18.

Monderer, D., Samet, D., 1989. Approximating common knowledge with common beliefs. Games Econ. Behav. 1, 170-190.

Morris, S., Shin, H.S., 1997. Approximate common knowledge and coordination: recent lessons from game theory. J. Logic Lang. and Info. 6, 171-190.

Nöldeke, G., Samuelson, L., 1994. Learning to signal in markets. working paper, Princeton University, Department of Economics, and University of Wisconsin, Department of Economics.

Nöldeke, G., Samuelson, L., 1997. A dynamic model of equilibrium selection in signaling markets. J. Econ. Theory 73, 118-156.

Rubinstein, A., 1989. The electronic mail game : strategic behavior under "almost common knowledge". Amer. Econ. Rev. 79, 385-391.

Selten, R., 1980. A note on evolutionary stable strategies in asymmetric animal contests. J. Theor. Biol. 84, 93-101.

Swinkels, J., 1992. Evolutionary stability with equilibrium entrants. J. Econ. Theory 57, 306-332.

Thomas, B., 1985. On evolutionary stable sets. J. Math. Biol. 22, 105-115. 